Revue internationale P.M.E.

Économie et gestion de la petite et moyenne entreprise

\title{
Petites et moyennes entreprises à forte croissance et emploi dans le secteur manufacturier grec
}

\section{George Zaralis}

Volume 14, numéro 3-4, 2001

URI : https://id.erudit.org/iderudit/1008703ar

DOI : https://doi.org/10.7202/1008703ar

Aller au sommaire du numéro

Éditeur(s)

Presses de l'Université du Québec

ISSN

0776-5436 (imprimé)

1918-9699 (numérique)

Découvrir la revue

Citer cette note

Zaralis, G. (2001). Petites et moyennes entreprises à forte croissance et emploi dans le secteur manufacturier grec. Revue internationale P.M.E., 14(3-4),

211-239. https://doi.org/10.7202/1008703ar
Résumé de l'article

Cette étude a pour objet l'influence des PME à forte croissance sur la création d'emplois dans le secteur manufacturier grec. L'analyse empirique a été faite à partir de données provenant de recensements relevés au niveau d'établissements de plus de 20 salariés au cours de la période de cinq ans comprise entre 1992 et 1996. À partir de la croissance absolue, du taux de croissance et des indices de Birch et de Mustar, on a défini quatre groupes rassemblant les $10 \%$ de PME qui se situent en tête pour la croissance. Les résultats des estimations réalisées par la méthode des probits indiquent que la taille de l'entreprise, les dépenses d'innovation, les exportations, la rentabilité, l'emplacement et la croissance du secteur agissent sur la probabilité d'appartenir aux quatre différents groupes de PME à forte croissance.
Ce document est protégé par la loi sur le droit d'auteur. L'utilisation des services d’Érudit (y compris la reproduction) est assujettie à sa politique d'utilisation que vous pouvez consulter en ligne.

https://apropos.erudit.org/fr/usagers/politique-dutilisation/ 


\section{Petites et moyennes entreprises à forte croissance et emploi dans le secteur manufacturier grec}

George ZARALIS

Représentant permanent adjoint de la Grèce auprès de l'OCDE

\section{MOTS CLÉS}

\section{Petites et moyennes entreprises - Secteur manufacturier High-employment growth - Modèles de probit}

\section{RÉSUMÉ}

Cette étude a pour objet l'influence des PME à forte croissance sur la création d'emplois dans le secteur manufacturier grec. L'analyse empirique a été faite à partir de données provenant de recensements relevés au niveau d'établissements de plus de 20 salariés au cours de la période de cinq ans comprise entre 1992 et 1996. À partir de la croissance absolue, du taux de croissance et des indices de Birch et de Mustar, on a défini quatre groupes rassemblant les $10 \%$ de PME qui se situent en tête pour la croissance. Les résultats des estimations réalisées par la méthode des probits indiquent que la taille de l'entreprise, les dépenses d'innovation, les exportations, la rentabilité, l'emplacement et la croissance du secteur agissent sur la probabilité d'appartenir aux quatre différents groupes de PME à forte croissance.

\section{L'AUTEUR}

George Zaralis est titulaire d'une M.Sc. de I'Université de Londres (Royaume-Uni) et d'un doctorat en économie de l'Université Concordia (Canada). Il a enseigné l'économie dans les universités Concordia, Ryerson et de Windsor au Canada (1985-1993). Il est actuellement le représentant permanent adjoint de la Grèce auprès de l'OCDE. Son principal domaine de recherches concerne l'économie industrielle. Adresse: 15, Villa Said, 75116 Paris, France. Téléphone : 1450224 10. Télécopieur : 1450071 55. Courriel : <MEA.OOSA@wanadoo.fr>.

Je tiens à exprimer toute ma gratitude à $\mathrm{D}$. Serrelis pour les observations et les conseils qu'il a formulés au sujet de cette étude ainsi qu'à D. Andreou qui m'a grandement aidé dans mes recherches. 


\begin{abstract}
This paper examines the contribution of high growth SMEs to employment creation in the Greek manufacturing sector. The empirical analysis is conducted using establishment level census data of sizes larger than 20 employees for the five-year period 1996-1992. The study employs the absolute growth, growth rate, Birch and Mustar indices to select four corresponding groups consisting of the top ten percent high growth SMEs. Probit estimation results indicate that firm size, innovation, exports, profitability and industry growth influence the likelihood of firms to belong to the four different groups of high growth SMEs.
\end{abstract}

\title{
RESUMEN
}

Este estudio tiene por objecto la influencia de las PyMEs (Pequeñas y Medianas Empresas) con fuerte crecimiento sobre la creación de empleo en el sector manufacturero griego. El análisis empfrico se lleva a cabo con ayuda de datos provenientes de establecimientos de más de 20 asalariados durante el periodo de cinco años comprendidos entre 1992 y 1996. A partir del crecimiento absoluto, de la tasa de credimiento y de los indices de Birch y de Mustar, se han definido cuartro grupos que reúnen en 10 por ciento de las PyMEs que se sitúan en cabeza por su crecimiento. Los resultados de las evaluaciones por el método de los probits indican que el tamaño de las empresas, los gastos de innovación, las exportaciones, la rentabilidad, la ubicación, y el crecimiento del sector tienen una influencia sobre la probabilidad de pertenecer a los cuatro diferentes grupos de PyMEs con fuerte crecimiento.

\section{ZUSAMMENFASSUNG}

Diese Studie untersucht den Einfluss der stark wachsenden KMU auf die Beschäftigtengründung im Produktionssektor in Griechenland. Die empirische Analyse ist begleitet mit der Hilfe von Daten, die erhoben wurden bei 20 Angestellten während der Periode von 5 Jahren, zwischen 1992 und 1996.

Ausgehend vom absolutem Wachstum, der Wachstumsrate und den Indikatoren von Birch und Mustar, hat man vier Gruppen definiert die $10 \%$ der KMU umfassen. Diese Gruppen gehören zur Spitzengruppe der stark wachsenden Unternehmen. Die Ergebnisse der realisierten Schätzungen unterstreichen, dass die Grösse der Unternehmung, die Innovationsausgaben, die Exporte, die Rendite, Standort und das Wachstum der Branche auf die Wahrscheinlichkeit wirken, zu den vier verschiedenen Gruppen der stark wachsenden KUM zu gehören.

\section{Introduction}

L'intérêt des chercheurs pour le rôle des PME dans la création d'emploi a été stimulé ces dernières années par l'ampleur du chômage en Europe et sur d'autres continents, ainsi que par les résultats de plusieurs études indiquant que certains groupes de PME pouvaient contribuer sensiblement à la croissance de l'emploi (Birch, 1981, 1987 ; 
Storey, 1994; OCDE, 2001). Le présent document analyse les statistiques disponibles dans le but de définir la contribution des petites et moyennes entreprises à forte croissance (PMEfc) à la croissance globale de l'emploi dans le secteur manufacturier grec durant la période 1988-1992. Il tente également de répertorier les facteurs qui affectent la contribution des PMEfc à la croissance de l'emploi.

Les PME et PMEfc sont définies ${ }^{1}$ en fonction de leurs effectifs et l'analyse ne tient compte que de celles qui étaient en activité pendant toute la période considérée. On a utilisé des indicateurs absolus et relatifs pour classer et sélectionner les entreprises créatrices d'emplois qui se situent dans la tranche des $10 \%$ de tête (entreprises à forte croissance). Les classes de taille des entreprises de plus de 20 salariés et les branches d'activité sont clairement définies. Il n'a cependant pas été possible, en raison des lacunes des données, de distinguer entre les sociétés mères et les filiales et entre la croissance interne et la croissance due à des acquisitions.

En général, la variation de l'emploi dans un secteur n'est pas liée uniquement à l'expansion et au recul des entreprises permanentes, mais aussi à la création et à la disparition d'un certain nombre d'entreprises. La présente étude, consacrée uniquement aux entreprises permanentes, fait abstraction des effets sur l'emploi des entreprises nouvellement créées et disparues. Il est évident que pour donner une image plus exacte de la contribution des PME à la croissance de l'emploi, il faudrait réaliser une analyse complémentaire de la création d'emplois par les entreprises nouvelles et de la suppression d'emplois qu'entraînent les entreprises qui disparaissent.

Le document est organisé comme suit: la section 1 présente les données, les indicateurs de croissance et les classes de taille qui ont été utilisées. La section 2 donne les résultats empiriques concernant l'emploi et sa croissance dans toutes les entreprises de plus de 20 salariés de l'industrie manufacturière. La section 3 est consacrée aux caractéristiques de quatre groupes de PMEfc définis en fonction de quatre indicateurs de croissance différents. La définition des modèles de probits et les estimations de la probabilité de forte croissance qu'ils permettent de réaliser sont étudiées à la section 4. La dernière section fait la synthèse des principales conclusions.

\section{Données, indicateurs de croissance, classes de taille}

On a utilisé des données du Service national des statistiques de Grèce (SNSG) qui proviennent de recensements, couvrant le secteur manufacturier grec au niveau des établissements pour la période 1996-1992 et leur classification est fondée sur le

1. Cette étude se fonde sur la méthode générale proposée par le groupe de travail de l'OCDE sur les PME et décrite dans le document DSTI/IND/PME (97)9 dans le but de faciliter les comparaisons entre pays. Il faut cependant souligner que l'estimation qui a été faite dans cette étude de la contribution des PMEfc à l'emploi est influencée par la méthode adoptée et les indicateurs de croissance utilisés.

Revue internationale P.M.E., vol. 14, $\mathrm{n}^{\text {os }} 3-4,2001$ 
NACE REV1 de l'EUROSTAT. L'étude ne concerne que les établissements de plus de 20 salariés restés en activité pendant toute la période considérée. L'unité d'analyse est celle des établissements et il est généralement admis que la très grande majorité des entreprises grecques de ce secteur ne comporte qu'un seul établissement (Fotopoulos et Spence, 1998 ; Droucopoulos et Thomadakis, 1993).

Les indices de croissance de l'emploi utilisés sont les suivants:

Croissance absolue $=$ Emp1 - Emp0

Taux de croissance $=(E m p 1-$ Emp0 $) / E m p 0$

Indice de Birch $=($ Croissance absolue $) *($ Taux de croissance $)$

Indice de Mustar $=($ Emp1 - Emp0 $) *(E m p 1 /$ Emp0 $)$

où $\quad \mathrm{Emp0}=$ emploi pendant la période de référence

Emp1 = emploi à la fin de la période

De ces quatre indices, la croissance absolue semble le plus intéressant à utiliser lorsqu'on veut établir la contribution maximale des PMEfc à l'emploi total. En outre, les niveaux de croissance absolue les plus élevés sont en principe liés aux entreprises les plus grandes. En revanche, les taux de croissance élevés sont en principe associés à des PMEfc plus petites et à un nombre inférieur d'emplois totaux créés. L'utilisation des indices de Birch et de Mustar peut aboutir à une sélection plus équilibrée des PMEfc, en termes de taille, mais ces deux indices paraissent moins attrayants d'un point de vue intuitif et ne sont pas explicitement liés à une théorie de croissance des entreprises.

\subsection{Classes de taille}

Les établissements sont classés en fonction de leurs effectifs en 1988 ; les classes utilisées sont les suivantes :

$$
\begin{array}{ll}
20-49 \text { salariés } & 250-499 \text { salariés } \\
50-99 \text { salariés } & 500-1000 \text { salariés } \\
100-249 \text { salariés } & 1000 \text { et plus }
\end{array}
$$

Il s'agit d'une répartition couramment employée dans les études de ce type. Il faut cependant noter que des définitions différentes des classes de taille peuvent se traduire par des résultats différents pour ce qui concerne la variation nette et brute de l'emploi dans les différentes classes. C'est pourquoi les conclusions des sections 3 et 4 sont, dans une certaine mesure, subordonnées aux classes choisies.

La méthode appliquée pour définir la taille des entreprises par rapport à la période considérée et les classer peut, elle aussi, affecter les conclusions formulées sur la variation de l'emploi. Dans cette étude, on a utilisé la taille pendant la période de référence (plutôt que la taille moyenne sur une période donnée) pour répartir 
les entreprises selon les classes définies. Cette méthode correspond à une conception «schumpétérienne » du fonctionnement des entreprises et des secteurs qui a été critiqué, dans le cadre du débat sur le biais de la « régression à la moyenne », par des chercheurs qui pensent que les entreprises tendent à converger vers une sorte d'équilibre ou de profil de croissance à long terme ${ }^{2}$.

\section{Croissance de l'emploi : résultats empiriques}

\subsection{Emploi et répartition des taux de croissance en 1988, 1990 et 1992}

Le tableau 1 donne des informations générales sur les niveaux d'emploi observés en fonction de la taille de l'entreprise en 1992, 1994 et 1996. Ce tableau concerne des établissements en activité pendant toute la période de 1996 à 1992 et dont les effectifs étaient supérieurs à 20 personnes en 1992, 1994 et 1996. Le nombre total d'entreprises diffère en raison des effectifs de certaines entreprises qui ont pu passer au-dessus ou au-dessous de la barre des 20 salariés durant la période de référence. De même, les différences concernant l'emploi total correspondent à des gains ou à des pertes nettes d'emplois dans des entreprises de plus de 20 salariés et aux entrées et sorties d'entreprises de plus de 20 salariés.

TABLEAU 1

Emploi et nombre d'entreprises par classe de taille

\begin{tabular}{lrrrrrr}
\hline Taille de l'entreprise & \multicolumn{3}{c}{ Emploi } & \multicolumn{3}{c}{ Nombre d'entreprises } \\
\hline (Effectifs) & $\mathbf{1 9 9 2}$ & $\mathbf{1 9 9 4}$ & $\mathbf{1 9 9 6}$ & $\mathbf{1 9 9 2}$ & $\mathbf{1 9 9 4}$ & $\mathbf{1 9 9 6}$ \\
\hline $20-49$ & 42655 & 40077 & 37056 & 1360 & 1283 & 1203 \\
$50-99$ & 34144 & 32450 & 32434 & 498 & 467 & 469 \\
$100-249$ & 50319 & 48705 & 50208 & 329 & 319 & 322 \\
$250-499$ & 39324 & 36427 & 32891 & 113 & 107 & 94 \\
$500-999$ & 23219 & 26227 & 26544 & 34 & 38 & 38 \\
1000 et plus & 25554 & 19665 & 16961 & 16 & 12 & 11 \\
\hline Total & 215215 & 203551 & 196094 & 2350 & 2226 & 2137 \\
\hline
\end{tabular}

Les données indiquent que l'emploi total ainsi que le nombre total d'entreprises de plus de 20 personnes ont diminué pendant la période concernée.

2. Voir Davies, Haltiwanger et Schuh (1996), Carree et Klomp (1996), Davidsson, Lindmark et Olofsson (1998), Kirchhoff et Greene (1998) entre autres.

Revue internationale P.M.E., vol. 14, $\mathrm{n}^{\text {os }} 3-4,2001$ 
En pourcentage, on observe une baisse intermédiaire de l'emploi de 5,4\% de 1994 à 1992 et une baisse globale de l'ordre de 8,8 \% de 1996 à 1992. En ce qui concerne le nombre d'entreprises, les variations sont respectivement de 5,2\% pour 1994-1992 et de $9 \%$ pour 1996-1992.

Dans toutes les entreprises des différentes classes, l'emploi n'a cessé de baisser pendant toute la période considérée sauf dans les entreprises de 500 à 999 salariés. Les entreprises de ces classes représentaient 89,2\% de l'emploi en 1992 et $86,4 \%$ en 1996. Pour ce qui est des entreprises de 500 à 999 salariés, on observe un accroissement continu de l'emploi, les pourcentages correspondants étant de 10,7 et $13,5 \%$ en 1992 et 1996 respectivement.

La variation du nombre d'entreprises des différentes classes suit des tendances similaires pour les mêmes périodes. Dans ce cas, le nombre d'entreprises de 500 à 999 salariés présente une augmentation nette pour la période 1992-1996. Ces variations de l'emploi et du nombre d'entreprises correspondent aussi aux mouvements des entreprises entre les différentes classes.

Il est intéressant, au regard de l'évolution de l'emploi, de se pencher sur la répartition des indicateurs de croissance absolue et relative. Le tableau 2 donne la répartition des taux de croissance de l'emploi et des chiffres d'affaires pour 19941992, 1996-1994 et 1996-1992. Le tableau 3 montre la répartition de la variation absolue de l'emploi durant les mêmes périodes. Le nombre d'entreprises de plus de 20 salariés en activité de 1992 à 1996 est de 1884, leurs effectifs passant de 198781 à 187846 personnes (voir tableau 8). Comme l'indique le tableau 1, le nombre d'entreprises de plus de 20 salariés était de 2350 en 1992 et les effectifs, de 215215 personnes.

En ce qui à trait la répartition du taux de croissance de l'emploi en 1996-1992 (tableau 2), la médiane correspondante est égale à - $11 \%$, tandis que le taux de croissance requis pour qu'une entreprise appartienne à la catégorie des entreprises à forte croissance est supérieur à $31 \%$. On constate aussi que, concernant la répartition de la variation absolue de l'emploi durant la même période (tableau 3), la médiane est égale à -6 salariés et qu'une entreprise doit accroître ses effectifs de 17 personnes pour appartenir aux $10 \%$ se situant en tête du groupe des entreprises créatrices d'emplois.

\subsection{Taille de l'entreprise, gains et pertes d'emplois}

La section précédente vise des indicateurs nets de l'emploi qui ne donnent cependant pas suffisamment de précisions sur le phénomène de la création et de la suppression d'emplois. On trouvera ci-après des éléments empiriques sur les variations brutes, positives ou négatives, de l'emploi, selon la taille des entreprises. 
TABLEAU 2

Répartition des taux de croissance de l'emploi et du chiffre d'affaires

\begin{tabular}{|c|c|c|c|c|c|c|}
\hline \multirow[b]{2}{*}{ PERCENTILE } & \multicolumn{3}{|c|}{ EMPLOI } & \multicolumn{3}{|c|}{ CHIFFRE D'AFFAIRES } \\
\hline & 1994-1992 & 1996-1994 & 1996-1992 & 1994-1992 & 1996-1994 & 1996-1992 \\
\hline $1 \%$ & $-0,68$ & $-0,73$ & $-0,81$ & $-0,73$ & $-0,80$ & $-0,82$ \\
\hline $5 \%$ & $-0,45$ & $-0,48$ & $-0,57$ & $-0,45$ & $-0,47$ & $-0,53$ \\
\hline $10 \%$ & $-0,32$ & $-0,32$ & $-0,93$ & $-0,24$ & $-0,27$ & $-0,28$ \\
\hline $25 \%$ & $-0,19$ & $-0,19$ & $-0,28$ & $-0,06$ & $-0,10$ & $-0,05$ \\
\hline $50 \%$ & $-0,06$ & $-0,07$ & $-0,11$ & 0,08 & 0,05 & 0,19 \\
\hline $75 \%$ & 0,05 & 0,04 & 0,06 & 0,30 & 0,24 & 0,55 \\
\hline $90 \%$ & 0,22 & 0,18 & 0,31 & 0,57 & 0,49 & 1,03 \\
\hline $95 \%$ & 0,42 & 0,39 & 0,61 & 0,89 & 0,78 & 1,57 \\
\hline $99 \%$ & 0,77 & 0,70 & 1,13 & 1,63 & 1,47 & 3,02 \\
\hline Moyenne & 0,03 & 0,02 & 0,03 & 0,30 & 0,21 & 0,56 \\
\hline $\begin{array}{l}\text { Nombre } \\
\text { d'observations }\end{array}$ & 1884 & 1884 & 1884 & 1884 & 1884 & 1884 \\
\hline
\end{tabular}

TABLEAU 3

Répartition de la variation absolue de l'emploi

\begin{tabular}{lccc}
\hline PERCENTILE & $\mathbf{1 9 9 4 - 1 9 9 2}$ & $\mathbf{1 9 9 6 - 1 9 9 4}$ & $\mathbf{1 9 9 6 - 1 9 9 2}$ \\
\hline $1 \%$ & $-213,50$ & $-266,83$ & $-393,33$ \\
$5 \%$ & $-81,48$ & $-73,32$ & $-118,80$ \\
$10 \%$ & $-36,01$ & $-32,24$ & $-55,94$ \\
$25 \%$ & $-15,33$ & $-13,83$ & $-23,77$ \\
$50 \%$ & $-3,64$ & $-3,65$ & $-6,16$ \\
$75 \%$ & 2,49 & 1,98 & 2,97 \\
$90 \%$ & 11,50 & 11,29 & 16,75 \\
$95 \%$ & 29,73 & 29,98 & 39,83 \\
$99 \%$ & 62,32 & 55,33 & - \\
\hline Moyenne & $-2,98$ & $-2,82$ & 18,80 \\
\hline Nombre d'observations & 1884 & 1884 & 1884 \\
\hline
\end{tabular}

Le nombre d'entreprises est d'abord indiqué par classe de taille et classe de taux de croissance de l'emploi au tableau 4, puis par classe de taille et classe de variation absolue de l'emploi au tableau 5.

Revue internationale P.M.E., vol. 14, $\mathrm{n}^{\text {os }} 3-4,2001$ 


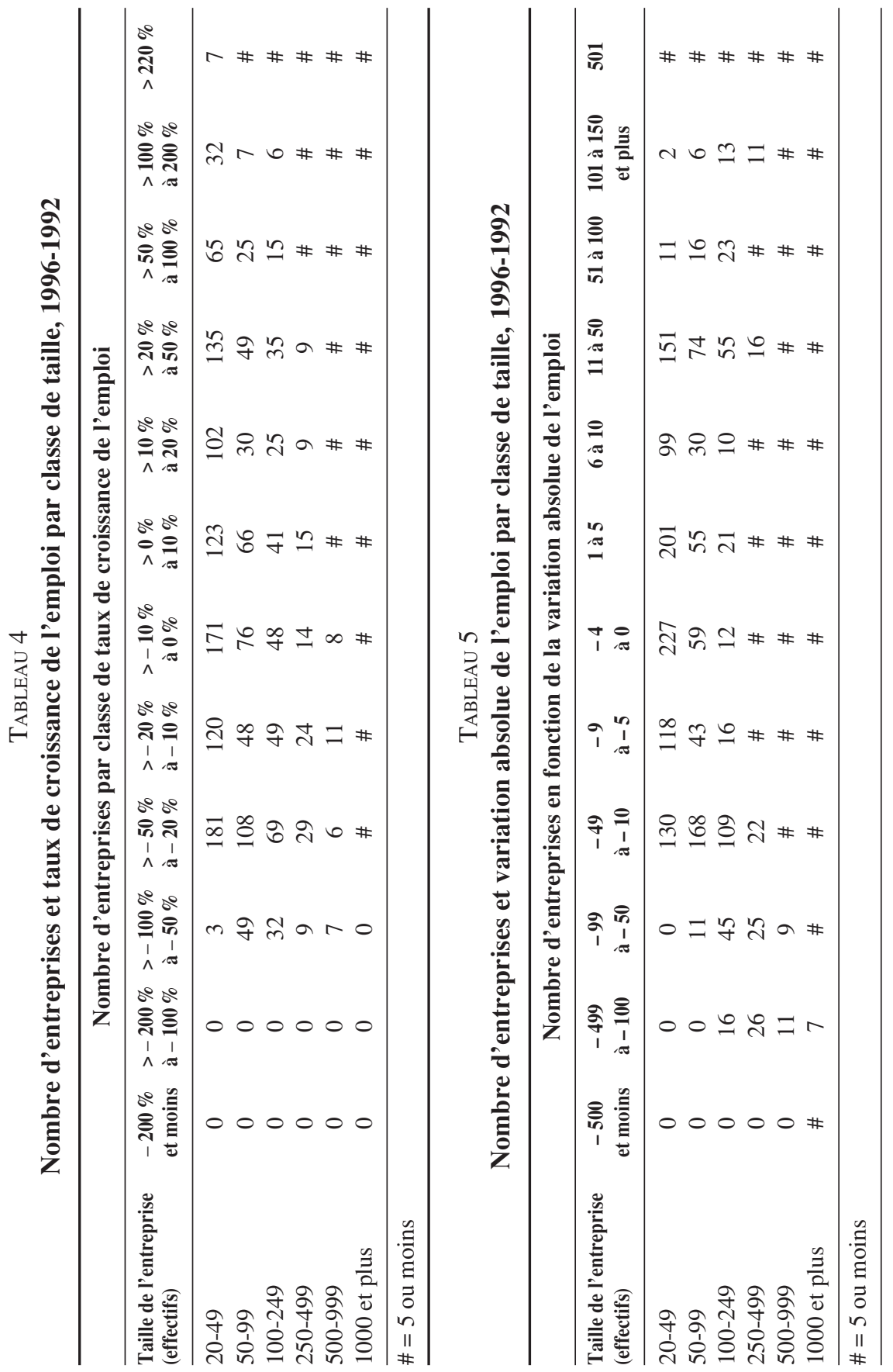

Revue internationale P.M.E., vol. 14, nos 3-4, 2001 
Le tableau 6 indique la variation absolue de l'emploi par classe de taille et par classe de variation absolue de l'emploi. Il donne une idée plus précise de la croissance de l'emploi et du nombre d'entreprises concernées, repris en termes de nombre d'entreprises en croissance et d'autres entreprises, et de variation positive et négative de l'emploi, au tableau 7 et au tableau 8 respectivement.

Le tableau 7 montre que, sur un total de 1884 entreprises, 816, soit 43,3\%, sont en croissance et que 1068 , soit $56,7 \%$, ne le sont pas. Sur ces 816 entreprises en croissance, presque $80 \%$ ont des effectifs compris entre 20 et 99 salariés. Parallèlement, $74 \%$ environ des entreprises qui ne sont pas en croissance font partie de ces deux premières classes de taille. En outre, le nombre des entreprises en croissance et des autres entreprises diminue selon une fonction monotone dans les classes de taille élevées, tandis que le nombre d'entreprises qui ne sont pas en croissance dépasse celui des entreprises en croissance dans toutes les classes de taille. Le tableau 8 présente les variations absolues de l'emploi et permet d'observer une baisse générale de l'emploi net de 10935 salariés (soit 5,5\%), due à la création de 20366 emplois bruts (10,2\%) et à la suppression de 31301 emplois bruts (15,7\%).

La première classe de taille (entreprises de 20 à 49 salariés) est la seule qui enregistre une variation nette positive de l'emploi, égale à 2523 salariés.

Les gains et pertes bruts d'emplois varient de façon irrégulière d'une classe de taille à l'autre, des gains bruts relativement élevés étant observés dans les trois classes inférieures $(27,2 \%, 23,5 \%$ et $28,6 \%)$, c'est-à-dire $79,3 \%$, tandis que les pertes brutes d'emploi sont plus nombreuses dans les deux classes moyennes (26,7 \% et $23,5 \%$ ) qui s'élèvent à $52,2 \%$. Le tableau 8 indique aussi que des gains et des pertes bruts sont enregistrés dans toutes les classes de taille pendant la période considérée.

\subsection{Branche d'activité, gains et pertes d'emploi}

Enfin, le tableau 9 donne des informations sur la croissance de l'emploi par branche d'activité à deux chiffres de l'industrie manufacturière. Au tableau 1 de l'annexe I figurent les codes de classification des activités économiques (CAE) et les activités correspondantes.

Le tableau 9 montre que sept branches d'activité ont connu une variation positive de l'emploi. La fabrication de machines électriques (CAE = 31), l'imprimerie et les activités connexes $(\mathrm{CAE}=22)$ enregistrent une croissance nette d'emploi de 36,2\% (803 salariés) et 29,9\% (664 salariés) respectivement. Les cinq autres branches d'activité, à savoir la fabrication de machines et appareils $(\mathrm{CAE}=29)$, la fabrication de matériels de bureau et d'ordinateurs $(\mathrm{CAE}=30)$, la fabrication d'appareils et de matériels électriques pour la radio, la télévision et les communications $(\mathrm{CAE}=32)$, la fabrication d'appareils médicaux et optiques et de 


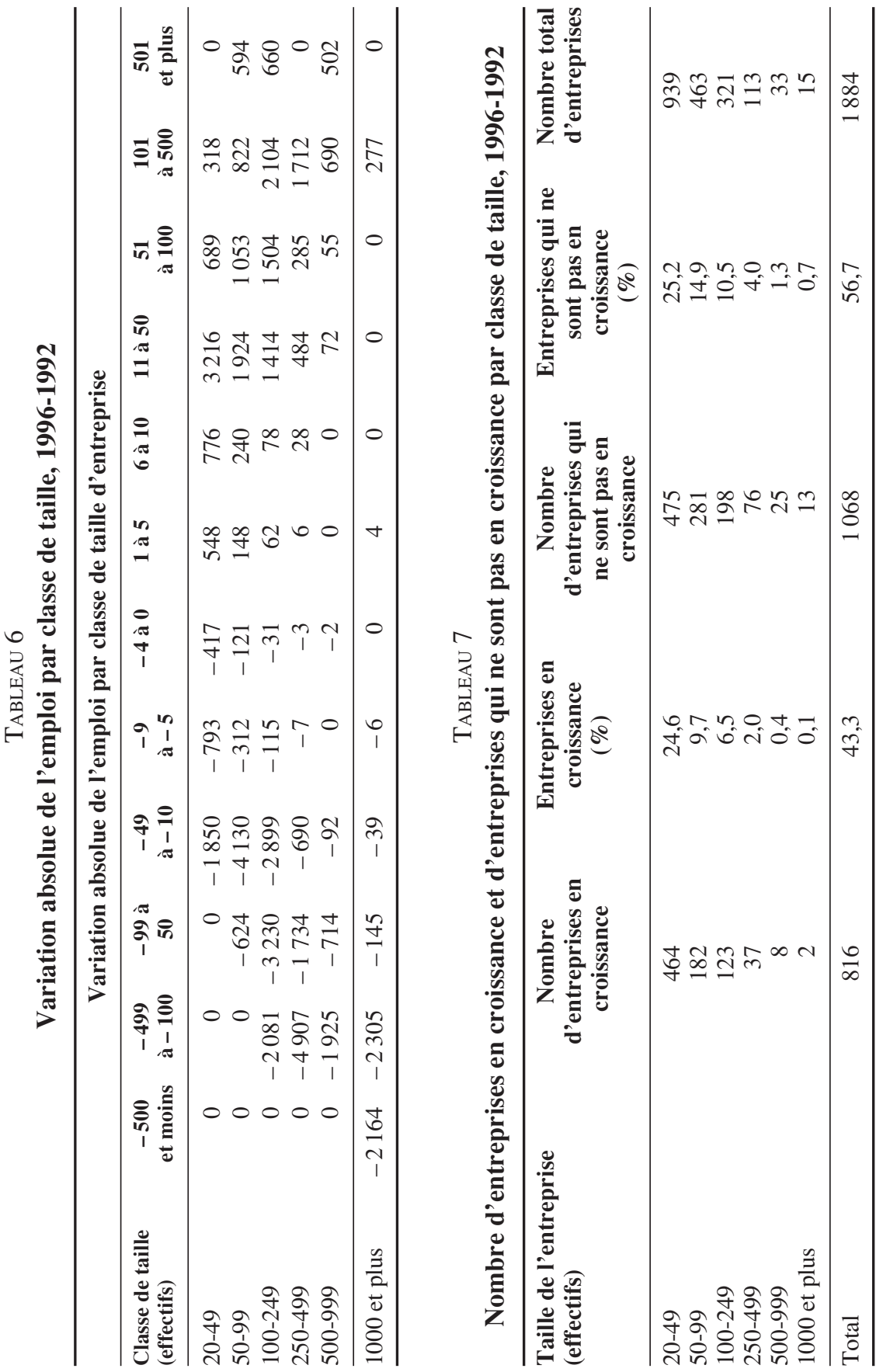

Revue internationale P.M.E., vol. 14, n ${ }^{\text {os }} 3-4,2001$ 


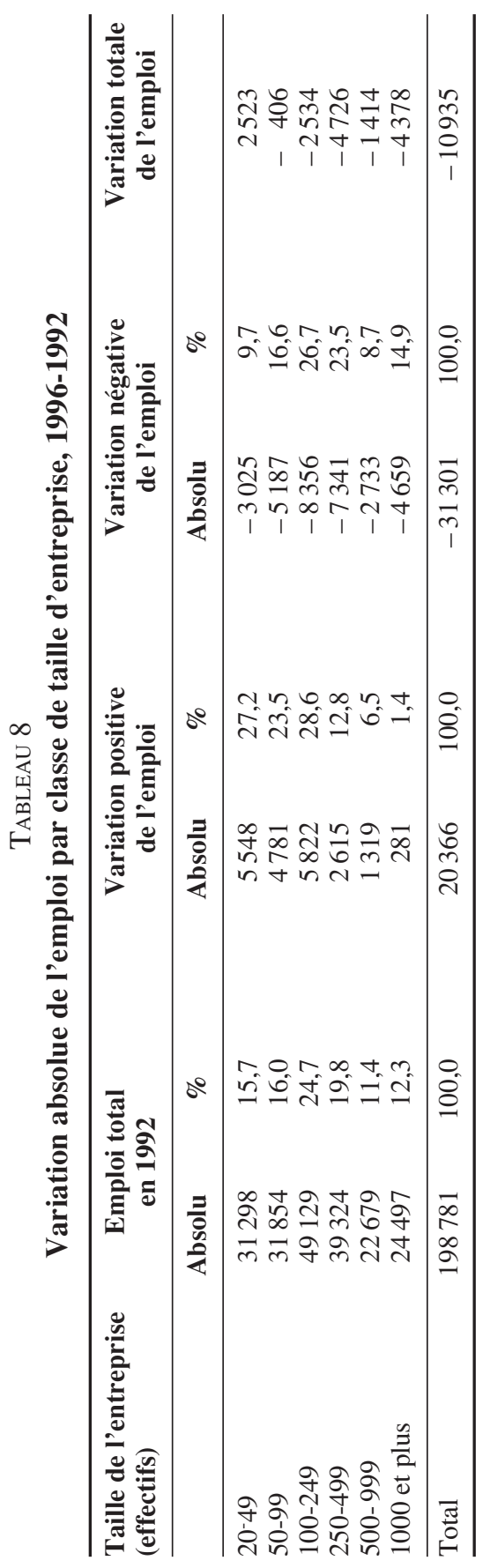

Revue internationale P.M.E., vol. 14, $\mathrm{n}^{\text {os }}$ 3-4, 2001 


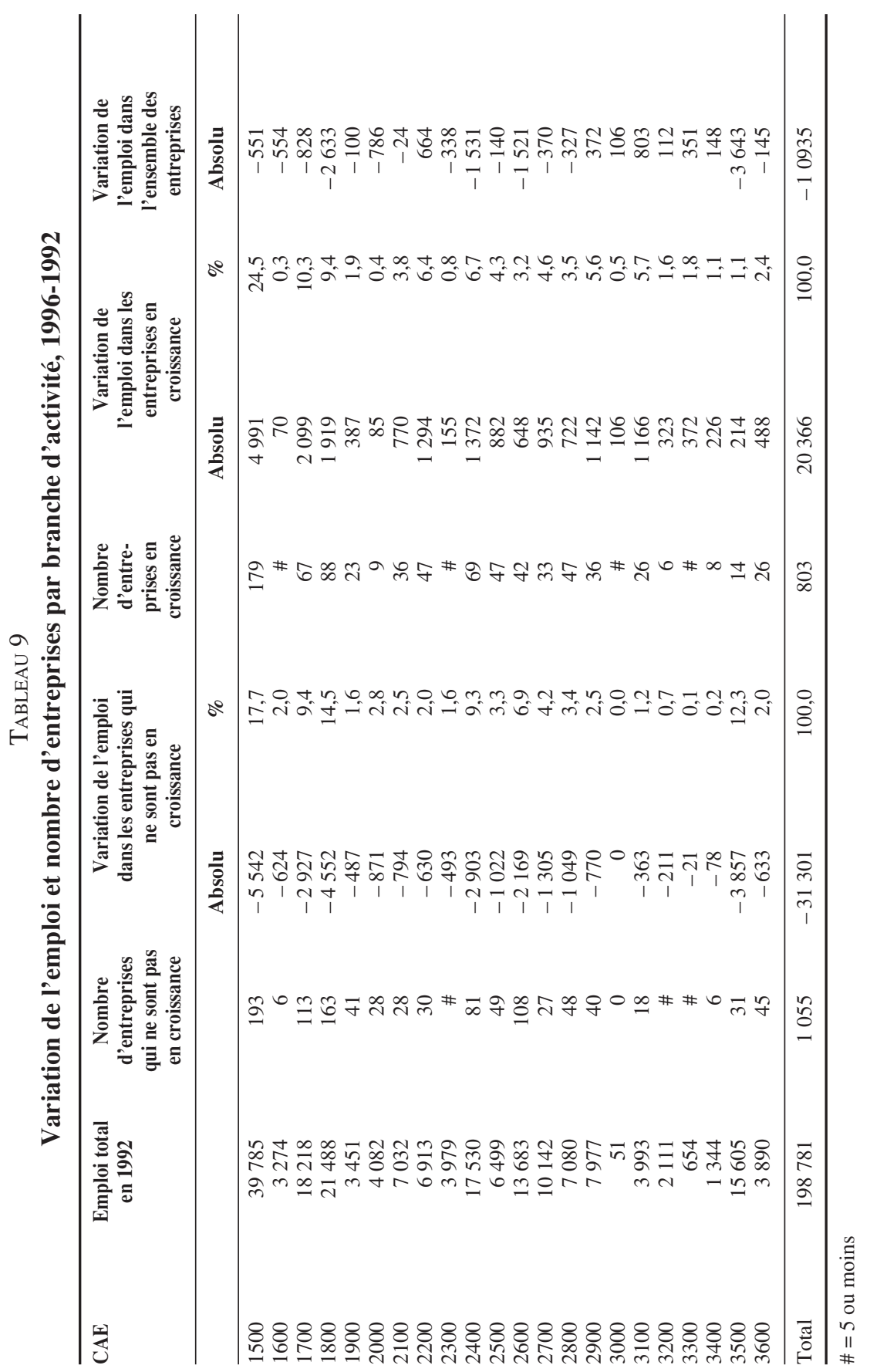

Revue internationale P.M.E., vol. 14, $\mathrm{n}^{\text {os }} 3-4,2001$ 
produits de laboratoire $(\mathrm{CAE}=33)$ et l'industrie automobile $(\mathrm{CAE}=34)$ enregistrent des taux de croissance compris entre $4,7 \%$ et $16,7 \%$. Les plus forts taux de contribution à l'emploi (plus de $10 \%$ ) des entreprises en croissance sont observés dans les branches des produits alimentaires $(\mathrm{CAE}=15)$ et du textile $(\mathrm{CAE}=17)$.

En outre, les contributions à l'emploi varient dans cinq branches entre $5 \%$ et $10 \%$. Dans les 10 autres branches, les entreprises en croissance contribuent pour moins de $5 \%$ à l'emploi. L'incidence négative des autres entreprises sur l'emploi est la plus élevée (plus de $10 \%$ ) dans les branches des produits alimentaires $(\mathrm{CAE}=15)$, des vêtements, accessoires d'habillement et fourrures $(\mathrm{CAE}=18)$ et dans les autres branches de transport $(\mathrm{CAE}=35)$.

Trois branches ont une incidence négative plus faible (entre $5 \%$ et $10 \%$ ) sur l'emploi. Dans 16 autres secteurs, l'effet négatif exercé sur l'emploi par les entreprises qui ne sont pas en croissance est inférieur à $5 \%$. Il convient de remarquer que les branches qui enregistrent les gains d'emploi les plus élevés sont aussi celles qui connaissent les pertes brutes les plus fortes. Il s'agit de celles des produits alimentaires $(\mathrm{CAE}=15)$, du textile $(\mathrm{CAE}=17)$ et des vêtements, accessoires d'habillement et fourrures $(\mathrm{CAE}=18)$. Signalons également qu'en général toutes les branches d'activité enregistrent à la fois des gains et des pertes bruts d'emplois.

\section{Petites et moyennes entreprises à forte croissance (PMEfc) : les $10 \%$ se situant en tête}

On s'est servi dans cette section des quatre indices de croissance de l'emploi définis dans la section 2 pour sélectionner quatre groupes d'entreprises à forte croissance parmi 1836 entreprises de 20 à 500 salariés. Ces indices sont les suivants : la croissance absolue, le taux de croissance, l'indice de Birch et l'indice de Mustar.

\subsection{Taille de l'entreprise et forte croissance}

Les tableaux 10 et 11 donnent les résultats de la sélection des groupes d'entreprises à forte croissance par classe de taille.

Comme on pouvait s'y attendre, l'indicateur de la croissance absolue permet de distinguer la plus forte (la plus faible) proportion d'entreprises appartenant à la

classe de taille la plus élevée (la plus basse), proportion égale à 10,4\% (27,3\%). C'est l'inverse qui se produit lorsqu'on utilise le taux de croissance: les classes correspondant aux effectifs les plus faibles et les plus importants rassemblent respectivement $63,4 \%$ et $1,6 \%$ des entreprises à forte croissance. L'incidence de la taille de l'entreprise s'atténue lorsqu'on utilise les indices de Birch et de Mustar; l'indice de Mustar aboutit à un «biais » relativement plus élevé en faveur des entreprises de plus grande taille. Trois des quatre méthodes de sélection indiquent un accroissement

Revue internationale P.M.E., vol. 14, nos 3-4, 2001 


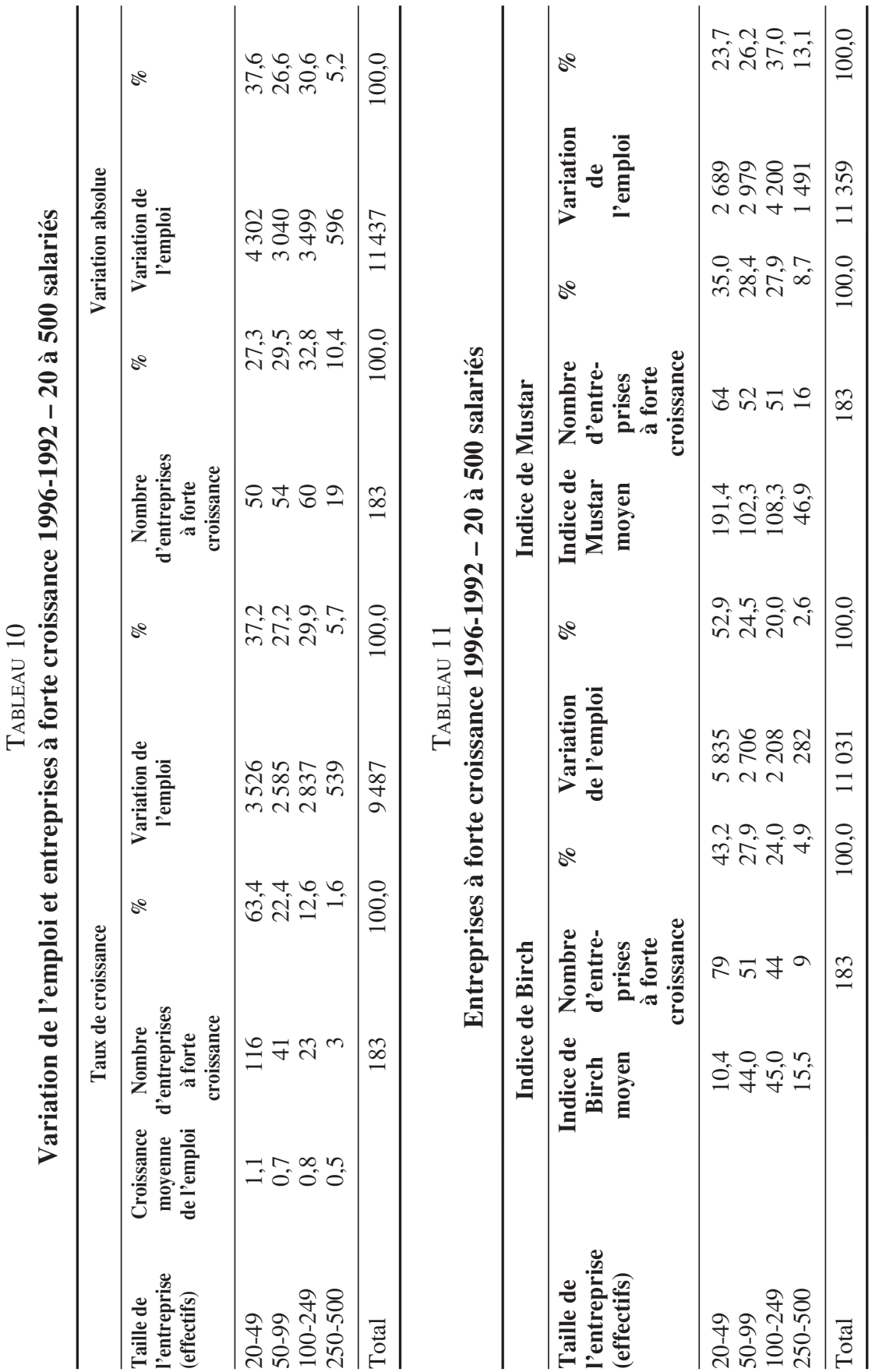

Revue internationale P.M.E., vol. 14, $\mathrm{n}^{\text {os }} 3-4,2001$ 
correspondant de l'emploi compris entre 11031 et 11437 . Ces chiffres absolus représentent $54,1 \%$ et $56,1 \%$ de l'emploi total créé par les entreprises «permanentes », y compris celles dont les effectifs dépassent 500 personnes. Lorsqu'on se sert de l'indicateur du taux de croissance, l'accroissement de l'emploi n'atteint que 9487 , soit $46,5 \%$ du total des emplois créés dans l'industrie manufacturière.

\subsection{Branches d'activité et entreprises à forte croissance}

On peut aussi chercher à déterminer dans quelles branches d'activité se trouvent les PMEfc. Les tableaux 12 et 13 indiquent qu'elles sont présentes dans presque toutes les branches. Seule la branche de la fabrication d'appareils et matériels pour la radio, la télévision et les communications $(\mathrm{CAE}=32)$ ne comptent pas d'entreprises à forte croissance. On trouve un pourcentage relativement plus élevé de PMEfc (plus de $9 \%)$ dans les branches des produits alimentaires $(\mathrm{CAE}=15)$ et des vêtements, accessoires d'habillement et fourrures (CAE $=18)$, indépendamment de l'indice de croissance utilisé. Il est intéressant de noter qu'il s'agit aussi des branches (y compris la branche des produits du textile $(\mathrm{CAE}=17)$ ) dont la contribution brute à l'emploi est la plus élevée. En outre, le pourcentage de PMEfc présentes dans ces trois branches est d'au moins $46 \%$ du total dans tous les cas ${ }^{3}$.

\subsection{Part des exportations dans les entreprises à forte croissance}

Pour obtenir la contribution des PMEfc aux exportations, on a calculé le nombre de PMEfc (N) et le rapport du nombre de PMEfc au nombre total de PME (R) en fonction de différentes classes de part des exportations (EXS), ce qui a donné les résultats suivants selon trois indices de croissance différents; à partir de l'indice de croissance absolue, $\mathrm{N}=67, \mathrm{R}=7,4 \%$ lorsque $\mathrm{EXS}=0 ; \mathrm{N}=68, \mathrm{R}=11,3 \%$ lorsque $0<\mathrm{EXS}<0,25 ; \mathrm{N}=17, \mathrm{R}=15,8 \%$ lorsque $0,25 \leq \mathrm{EXS}<0,50$; et $\mathrm{N}=31, \mathrm{R}=13,8 \%$ lorsque $0,50 \leq \mathrm{EXS}$.

Pour les mêmes classes de part des exportations, on obtient avec l'indice de Mustar les résultats suivants :

$$
\mathrm{N}=76, \mathrm{R}=8,4 \% ; \mathrm{N}=72, \mathrm{R}=12 \% ; \mathrm{N}=18, \mathrm{R}=16,8 \% ; \mathrm{N}=36, \mathrm{R}=16 \% \text {. }
$$

Dans les deux cas qui précèdent, le pourcentage de PMEfc dans les PME s'accroît pour les trois premières classes à mesure que la part des exportations augmente. Lorsqu'on utilise l'indice du taux de croissance, R montre une augmentation continue à mesure que la part des exportations augmente, à savoir :

3. Les tableaux 3, 4 et 5 de l'annexe I reproduisent les tableaux 9, 12 et 13 en utilisant la classification précédente afin de permettre aux lecteurs de procéder aux comparaisons entre les résultats de cette étude et d'une étude similaire (Zaralis, 1999) concernant la période 1992-1988.

Revue internationale P.M.E., vol. 14, $\mathrm{n}^{\text {os }} 3-4,2001$ 


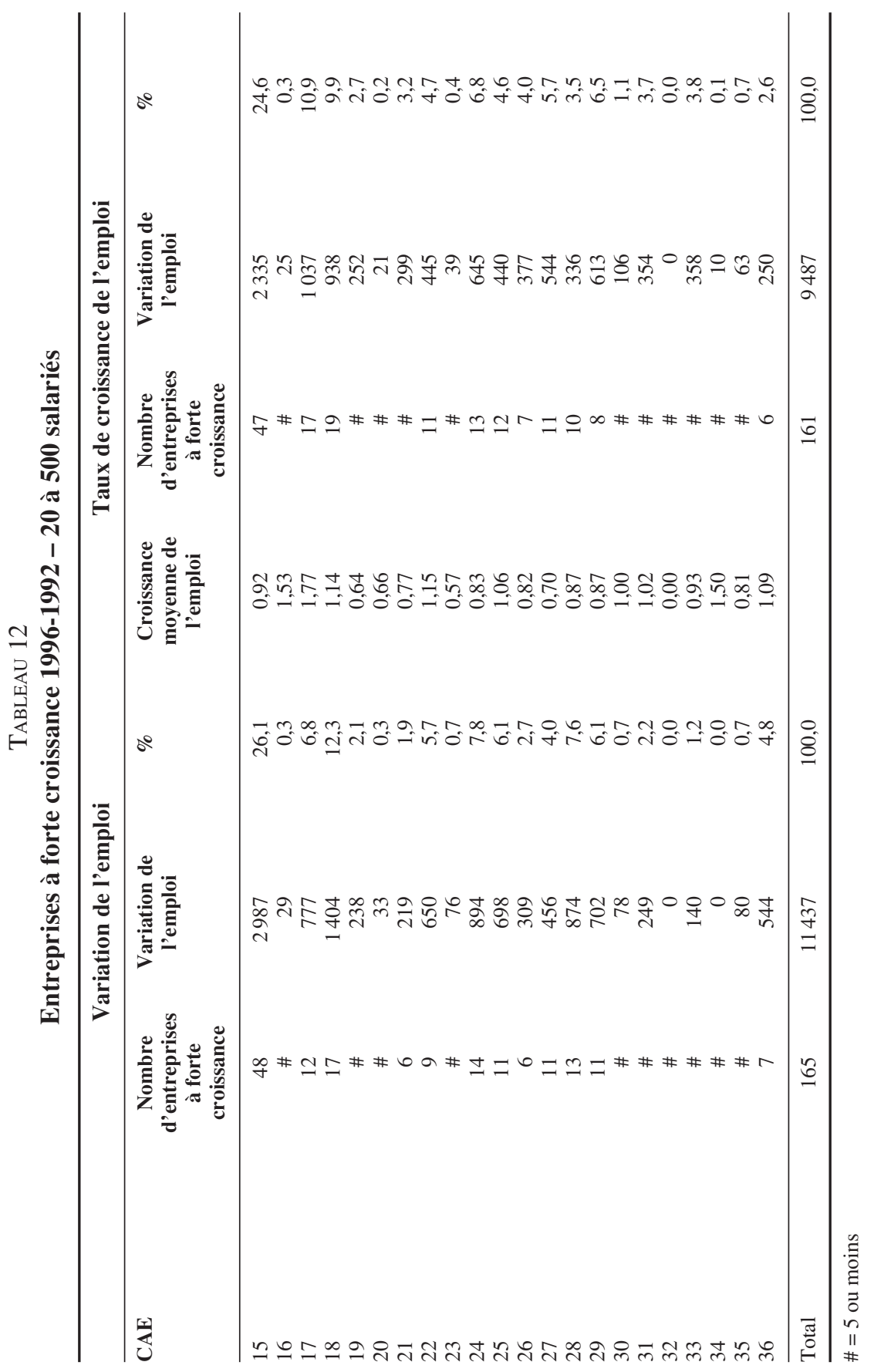

Revue internationale P.M.E., vol. 14, n ${ }^{\text {os }} 3-4,2001$ 


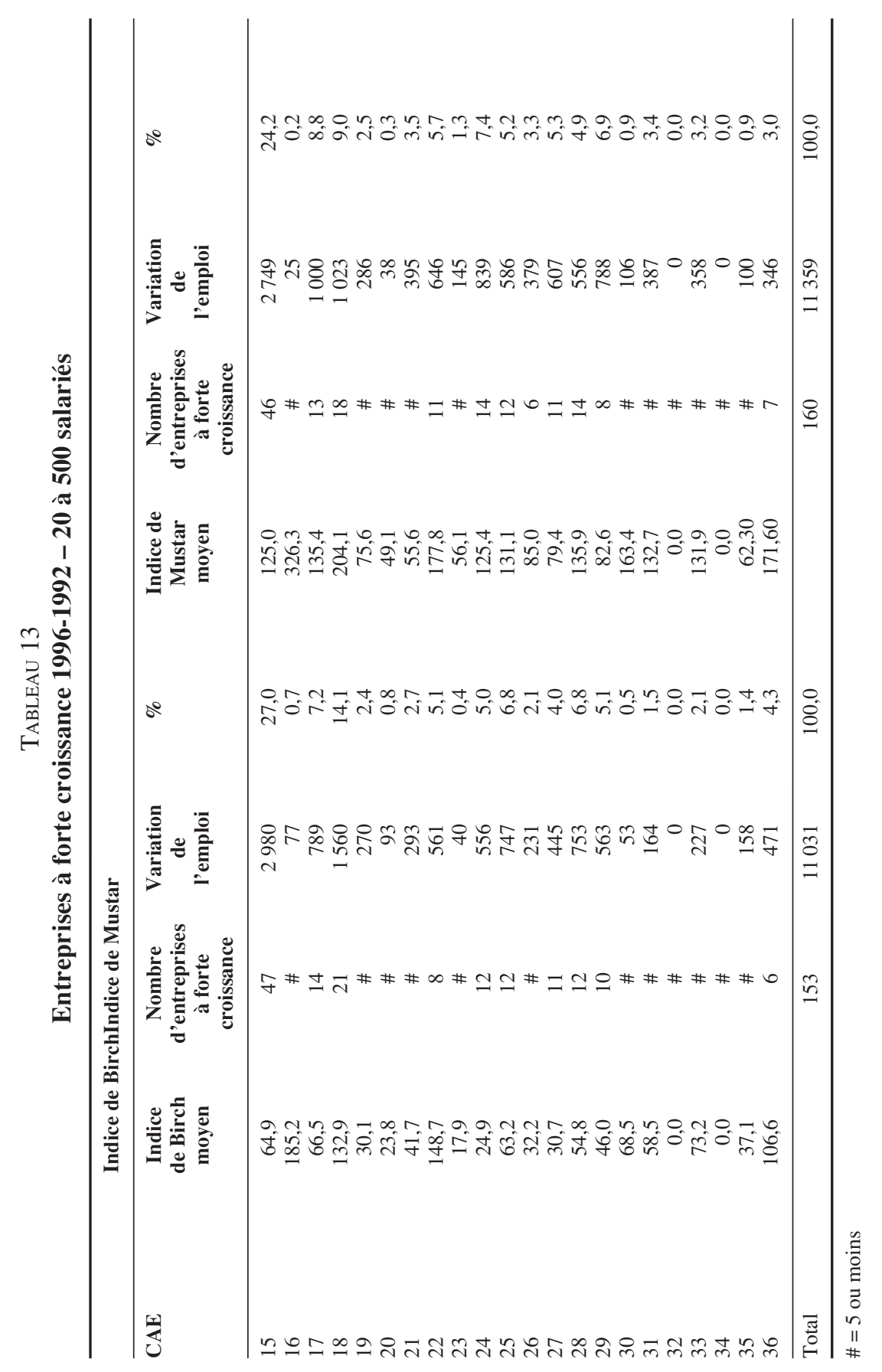

Revue internationale P.M.E., vol. 14, $\mathrm{n}^{\text {os }}$ 3-4, 2001 
$\mathrm{N}=78, \mathrm{R}=8,6 \% ; \mathrm{N}=55, \mathrm{R}=9,2 \% ; \mathrm{N}=16, \mathrm{R}=14,9 \% ; \mathrm{N}=34, \mathrm{R}=15,1 \%$.

Donc, dans les classes de part des exportations $\mathrm{EXS}=0$, le $\mathrm{R}$ varie entre 7,4\% et $8,6 \%$, tandis que dans les classes positives, le R est plus élevé et varie entre 9,2\% et 16,8 \%. La présence moyenne (R moyen) des PMEfc est 13,6\%, 14,9\% et 13,1\% selon l'indice de croissance absolue, l'indice de Mustar et l'indice du taux de croissance respectivement.

\section{Modèles de probits}

\subsection{Définition}

De nombreuses études empiriques portent sur les facteurs qui affectent la croissance des entreprises. Certaines analysent la relation entre la croissance et les caractéristiques des entreprises, à savoir la taille et l'âge, et examinent la validité de la théorie de Gibrat (Evans, 1987 a et b; Hall, 1987; Dunne et Hughes, 1994). D'autres travaux empiriques introduisent dans l'analyse des variables relatives aux caractéristiques de l'industrie, des dirigeants, etc., qui peuvent affecter la croissance des entreprises (Audretsch, 1995 ; Dunne, Roberts et Samuelson, 1989). En outre, d'autres études examinent la croissance des sous-ensembles spécifiques d'entreprises, comme les nouveaux entrants (Audretsch, 1995, chap. 4), les petites entreprises (Storey, 1994, chap. 5) et les entreprises à forte croissance (Storey, 1997) par exemple. Ces travaux empiriques utilisent en général une grande diversité de variables explicatives au niveau de l'industrie et de l'entreprise, variables dont l'importance potentielle est déterminée par les théories économiques et de gestion correspondantes.

Cette section est consacrée à l'influence exercée par certains facteurs sur la croissance d'un sous-ensemble d'entreprises, les PMEfc. Il s'agit plus précisément d'estimer la probabilité de forte croissance des entreprises par la méthode du modèle de probits (Greene, 1997 : ch. 19). Les PMEfc sont définies comme les $10 \%$ de PME qui ont enregistré la plus forte croissance de l'emploi pendant la période 1996-1992, sur la base de trois critères différents : la croissance absolue, la croissance relative (simple variation en pourcentage) et l'indice de Mustar, appliqués à la période 1996-1992. On aboutit à trois vecteurs renvoyant aux trois variables dépendantes des modèles de probits, lesquelles prennent la valeur un ou zéro selon qu'il s'agit des PMEfc ou des autres PME.

La sélection des variables indépendantes a été faite sur la base des indications de travaux théoriques et empiriques sur la question, mais elle a été limitée par des problèmes de disponibilité des données nécessaires. 
Les variables indépendantes choisies concernent soit l'entreprise, soit le secteur. Au niveau de l'entreprise, les variables utilisées sont la taille, la rentabilité, l'intensité de capital, l'innovation, la publicité, la flexibilité de la main-d'œuvre, les exportations et l'emplacement. Les variables au niveau du secteur sont la taille efficace minimale et la croissance du secteur. Les arguments théoriques qui justifient l'utilisation de ces variables dans les modèles de probits ainsi que le signe prévu des coefficients correspondants sont exposés ci-après.

La taille de l'entreprise sert de variable de contrôle ; compte tenu de la définition des variables dépendantes et de la sélection correspondante de PMEfc, on s'attend à ce que la probabilité de forte croissance de l'entreprise, en pourcentage, diminue avec la taille. Avec le critère de la croissance absolue et l'indice de Mustar, on peut s'attendre à ce que l'inverse se produise.

Il est raisonnable de s'attendre à ce que la probabilité de forte croissance de l'entreprise soit en corrélation positive avec sa rentabilité. Il est sans doute plus facile pour les entreprises de se développer rapidement en utilisant les bénéfices non distribués si la rentabilité est élevée, toutes choses égales d'ailleurs.

Une entreprise dont la production est caractérisée par une plus forte intensité de capital est susceptible de répondre plus lentement à l'accroissement de la demande en ajustant ses effectifs, toutes choses égales d'ailleurs, de tels ajustements nécessitant des investissements assez importants de capitaux.

Les dépenses d'innovation influent sensiblement sur la croissance de l'emploi, bien que l'incidence globale de ce facteur reste incertaine. L'innovation peut en effet se traduire par des économies de main-d'œuvre de production qui agissent négativement sur la croissance de l'emploi. Elle peut aussi aboutir à des produits nouveaux ou meilleurs qui, en faisant progresser le chiffre d'affaires de l'entreprise, se traduisent par une plus forte croissance de l'emploi.

La publicité, par la diffusion d'informations sur l'existence et les caractéristiques du produit et l'action qu'elle exerce sur la réputation de l'entreprise, peut entraîner une amélioration du chiffre d'affaires, de la part de marché et de la croissance de l'emploi. Cependant, cet effet positif peut être atténué ou annulé par les campagnes de promotion des entreprises concurrentes dans le même secteur.

L'entreprise peut bénéficier d'une importante marge de manœuvre en matière de recrutement si une partie des contrats offerts sont à durée déterminée. Le recours à de tels contrats réduit les engagements à long terme, financiers et autres, et peut faciliter les nouveaux recrutements. La probabilité de forte croissance peut donc être en corrélation positive avec ce type de flexibilité de la main-d'œuvre.

Revue internationale P.M.E., vol. 14, nos 3-4, 2001 


\section{Les exportations sont susceptibles d'influer positivement sur la croissance de l'emploi.}

L'emplacement de l'entreprise peut lui procurer des avantages directs ou indirects favorisant une forte croissance. Il peut être plus facile pour les entreprises de se développer dans les agglomérations urbaines ou les zones suburbaines, qui offrent par rapport aux zones rurales un meilleur accès aux marchés des biens de production, à des ressources spécialisées plus diversifiées ainsi qu'à des services publics et privés, davantage de débouchés, de meilleures infrastructures et de plus nombreuses externalités positives. Il est évident que l'importance de ces facteurs diminue lorsqu'il existe des réseaux de transport modernes et que les distances entre zones urbaines et rurales restent faibles. Durant la période d'observation, deux lois sur le développement étaient en vigueur; elles avaient pour effet de favoriser l'établissement et le développement d'entreprises dans les provinces grecques, plutôt que dans la région d'Athènes et de Thessalonique où les incitations à l'investissement étaient faibles ou nulles. Une probabilité plus élevée de plus forte croissance de l'emploi pouvait donc être attendue en province.

Les entreprises sont plus susceptibles de se développer rapidement dans les secteurs en expansion, de façon à tirer parti des possibilités de profits, de s'assurer une part de marché ou de l'améliorer parfois, alors que les taux d'entrées s'accroissent. Dans la mesure où les taux de forte croissance sont observés dans des entreprises jeunes de taille sous-optimale, on pourrait s'attendre à ce que la probabilité de croissance rapide augmente avec la taille efficace minimale du secteur. En d'autres termes, ces entreprises seront contraintes de ralentir leur croissance, suivant la courbe LRAC de l'industrie, pour améliorer leurs coûts et réagir à la concurrence.

Les définitions des variables utilisées sont les suivantes:

Part des exportations (EXP) Rapport montant des exportations / chiffre d'affaires, 1992.

Marge prix-coût (MPC) (Valeur ajoutée - traitements - salaires) / chiffre d'affaires, 1992.

Taille (Taille)

Effectifs, 1992.

Intensité de capital (IK)

Rapport capital / chiffre d'affaires; en l'absence de données sur le capital, on s'est servi des dépenses de carburant et d'électricité.

Innovation (INNO)

Acquisition d'équipements nouveaux et de matériel étranger d'occasion / chiffre d'affaires. 
Flexibilité de la main-d'œuvre (FLX)

Importance de la publicité (PUB)

Croissance du secteur (CS)

Taille efficace minimale (TEM)

Emplacement (EMPL)
Rapport contrats à durée déterminée / effectifs totaux.

Rapport dépenses de publicité / chiffre d'affaires.

(Chiffre d'affaires 1996 - Chiffre d'affaires 1992) / chiffre d'affaires 1992.

Taille moyenne des établissements les plus grands représentant $50 \%$ des effectifs totaux du secteur, 1992.

Variable muette $=1$, si l'entreprise est située dans la région Athènes-Thessalonique.

La taille de l'échantillon a été déterminée en fonction de la disponibilité des variables explicatives utilisées pour les entreprises de 20 à 500 salariés. Le nombre d'observations a été de 1707, soit un peu moins que le nombre total d'entreprises permanentes de plus de 20 salariés en activité pendant la période 1996-1992 (1884 entreprises).

\subsection{Résultats des estimations de probits}

Les résultats de l'estimation réalisée à partir des trois modèles de probits figurent au tableau 14. La probabilité de forte croissance de l'entreprise, en termes de croissance relative (modèle 2), diminue avec la taille, conclusion qui correspond aux résultats économétriques obtenus dans plusieurs autres études similaires. Par ailleurs, la probabilité de forte croissance absolue (modèle 1) augmente avec la taille comme prévu. L'introduction de la taille sous forme logarithmique n'a pas modifié ces résultats sur le plan qualitatif, ni leur signification statistique d'un modèle à l'autre.

Les coefficients d'innovation et de croissance du secteur sont également significatifs sur le plan statistique, ce qui indique que la probabilité qu'une entreprise appartienne au groupe des entreprises à forte croissance augmente avec la croissance du secteur et les dépenses d'innovation de l'entreprise. L'incidence des exportations et de la rentabilité sur la probabilité de forte croissance est positive dans les trois modèles, mais n'est statistiquement significative que dans les modèles 1 et 3 . La publicité et la flexibilité de la main-d'œuvre sont associées à des coefficients positifs mais les $t$ correspondants sont assez faibles.

L'intensité de capital a l'incidence négative prévue sur la probabilité de forte croissance mais n'a pas de signification statistique dans le modèle 3. Le coefficient de taille efficace minimale est sans signification statistique dans aucun des trois modèles, peut-être parce que l'échantillon ne comporte pas d'entreprises jeunes et 


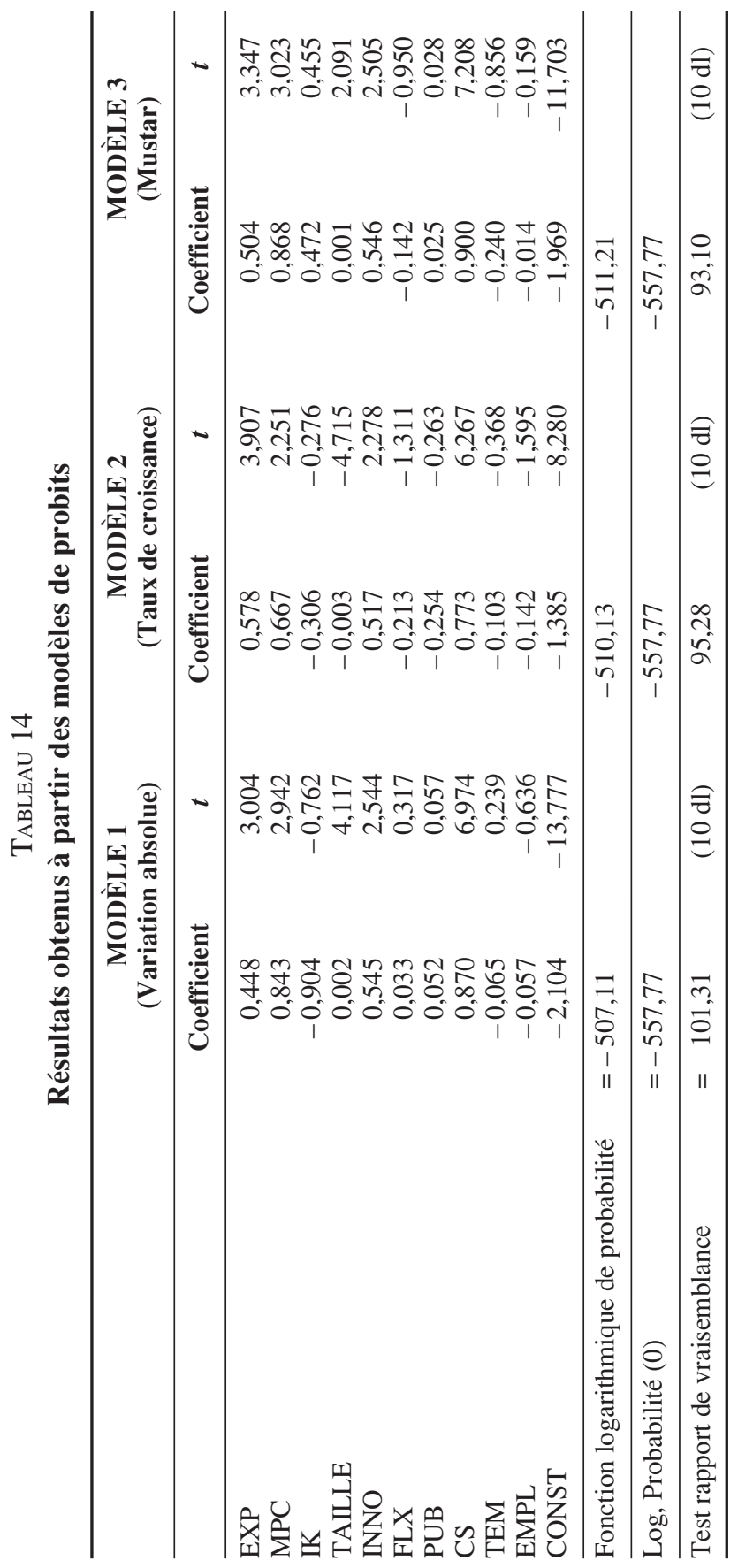

Revue internationale P.M.E., vol. 14, nos 3-4, 2001 
de taille sous-optimale. Enfin, le coefficient négatif de la variable muette d'emplacement indique que la probabilité d'appartenir aux entreprises à forte croissance augmente si l'entreprise se trouve en dehors de la région Athènes-Thessalonique.

On observe en général une similitude entre les signes des coefficients et la signification statistique des résultats des modèles 1 et 3, qui pourrait s'expliquer par le fait que les PMEfc de ces modèles sont plutôt de grandes entreprises, du fait des définitions des indicateurs de croissance utilisés (croissance absolue et indice de Mustar).

\section{Conclusion}

L'objet de cette étude était d'estimer la contribution à la création d'emplois des PME et des PME à forte croissance de l'industrie manufacturière grecque à l'aide de données de recensement correspondant à la période 1996-1992.

Il s'est produit pendant cette période une baisse générale de l'emploi et du nombre d'entreprises de plus de 20 salariés, bien qu'un accroissement de l'emploi et du nombre d'entreprises soit observé dans les entreprises de 500 à 999 salariés.

La répartition des variations absolues et relatives de l'emploi, associée dans tous les cas à des médianes à valeur négative, a été présentée pour les périodes 19941992, 1996-1994 et 1996-1992.

Le nombre des entreprises «permanentes », en croissance ou non, varie selon une fonction inverse dans les classes de taille de 1996 à 1992. Les gains et pertes bruts d'emploi correspondants suivent une évolution irrégulière. On observe dans toutes les classes des pertes nettes d'emplois, tandis que les classes les plus faibles sont associées à de plus forts gains nets d'emplois. En outre, des gains et des pertes bruts ont été observés dans toutes les classes de taille. Des gains nets d'emplois ont été enregistrés dans sept branches d'activité à deux chiffres. Les branches qui ont connu les gains bruts d'emploi les plus élevés étaient aussi celles qui ont subi les plus fortes pertes brutes. En plus, toutes les branches d'activité à deux chiffres sont associées à la fois à des gains et à des pertes bruts d'emploi.

La croissance absolue, le taux de croissance, l'indice de Mustar et l'indice de Birch ont servi à sélectionner quatre groupes de PMEfc (les $10 \%$ se situant en tête de l'ensemble des entreprises) pour la période 1996-1992. La composition de chaque groupe, en termes de taille de l'entreprise et de contribution à la création d'emplois, dépend de l'indice utilisé. Les PMEfc représentent 56,1\% et 46,5\% des totaux de nouveaux emplois si l'on utilise la croissance absolue et le taux de croissance respectivement. La contribution à l'emploi obtenue à partir des indices de Birch et de Mustar se trouve dans la même fourchette. Enfin, les PMEfc sont présentes dans presque toutes les branches d'activité à deux chiffres.

Revue internationale P.M.E., vol. 14, $\mathrm{n}^{\text {os }}$ 3-4, 2001 
L'étude portait aussi sur la définition et l'utilisation de modèles de probits permettant d'estimer la probabilité d'appartenir au groupe des $10 \%$ de PME à forte croissance. Les modèles comportaient un ensemble de 10 variables explicatives au niveau de l'entreprise et de l'industrie. La taille de l'entreprise a une incidence positive ou négative selon l'indice utilisé. La probabilité de forte croissance est en corrélation positive avec les dépenses d'innovation de l'entreprise et la croissance du secteur. De même, on observe une incidence positive et statistiquement significative des exportations et de la rentabilité de l'entreprise. L'intensité de capital de l'entreprise n'a qu'une incidence négative et statistiquement insignifiante. Tous les autres coefficients sont associés à des $t$ relativement faibles, sauf la variable de l'emplacement.

Il est évident que l'utilisation de variables supplémentaires comme l'âge de l'entreprise ou les caractéristiques des dirigeants aurait pu enrichir les résultats obtenus, mais ces éléments n'étaient malheureusement pas disponibles. 


\section{ANNEXE I}

\section{Tableau 1}

\begin{tabular}{ll}
\hline CAE & BRANCHES D'ACTIVITÉ À DEUX CHIFFRES \\
\hline 15 & Produits alimentaires et boissons \\
16 & Tabac \\
17 & Textile \\
18 & Fabrication de vêtements, d'accessoires d'habillement et de la fourrure \\
19 & Industries du cuir et des chaussures \\
20 & Articles en bois et en liège sauf meubles \\
21 & Papier \\
22 & Édition, imprimerie et activités connexes \\
23 & Produits dérivés du pétrole et du charbon \\
24 & Industrie chimique \\
25 & Industrie du caoutchouc et du plastique \\
26 & Fabrication de produits minéraux non métalliques \\
27 & Métallurgie \\
28 & Fabrication de produits métalliques finis sauf machines \\
29 & Fabrication de machines et d'appareils \\
30 & Fabrication de matériels de bureaux et d'ordinateurs \\
31 & Fabrication de machines, appareils et accessoires électriques \\
32 & Fabrication d'appareils et matériels pour la radio, la télévision et les communications \\
33 & Fabrication d'appareils médicaux, de laboratoire, etc. \\
34 & Industrie automobile \\
35 & Autres branches de transport \\
36 & Fabrication de meubles et autres industries \\
\hline
\end{tabular}

Tableau 2

\begin{tabular}{ll}
\hline CAE & BRANCHES D'ACTIVITÉ À DEUX CHIFFRES \\
\hline 20 & Produits alimentaires sauf boissons \\
21 & Boissons \\
22 & Tabac \\
23 & Textile \\
24 & Fabrication de chaussures, de vêtements et d'accessoires d'habillement \\
25 & Articles en bois et en liège sauf meubles \\
26 & Fabrication de meubles et d'articles de mobilier \\
27 & Papier \\
28 & Édition, imprimerie et activités connexes \\
29 & Industries de la fourrure et du cuir, sauf chaussures et vêtements \\
30 & Industrie du caoutchouc et du plastique \\
31 & Industrie chimique \\
32 & Produits dérivés du pétrole et du charbon \\
33 & Fabrication de produits minéraux non métalliques sauf dérivés du pétrole et du charbon \\
34 & Métallurgie \\
35 & Fabrication de produits métalliques finis sauf machines et matériels de transport \\
36 & Fabrication de machines et d'appareils sauf moteurs électriques et matériels de transport \\
37 & Fabrication de machines, appareils et accessoires électriques \\
38 & Matériels de transport \\
39 & Autres industries manufacturières
\end{tabular}

Revue internationale P.M.E., vol. 14, nos 3-4, 2001 


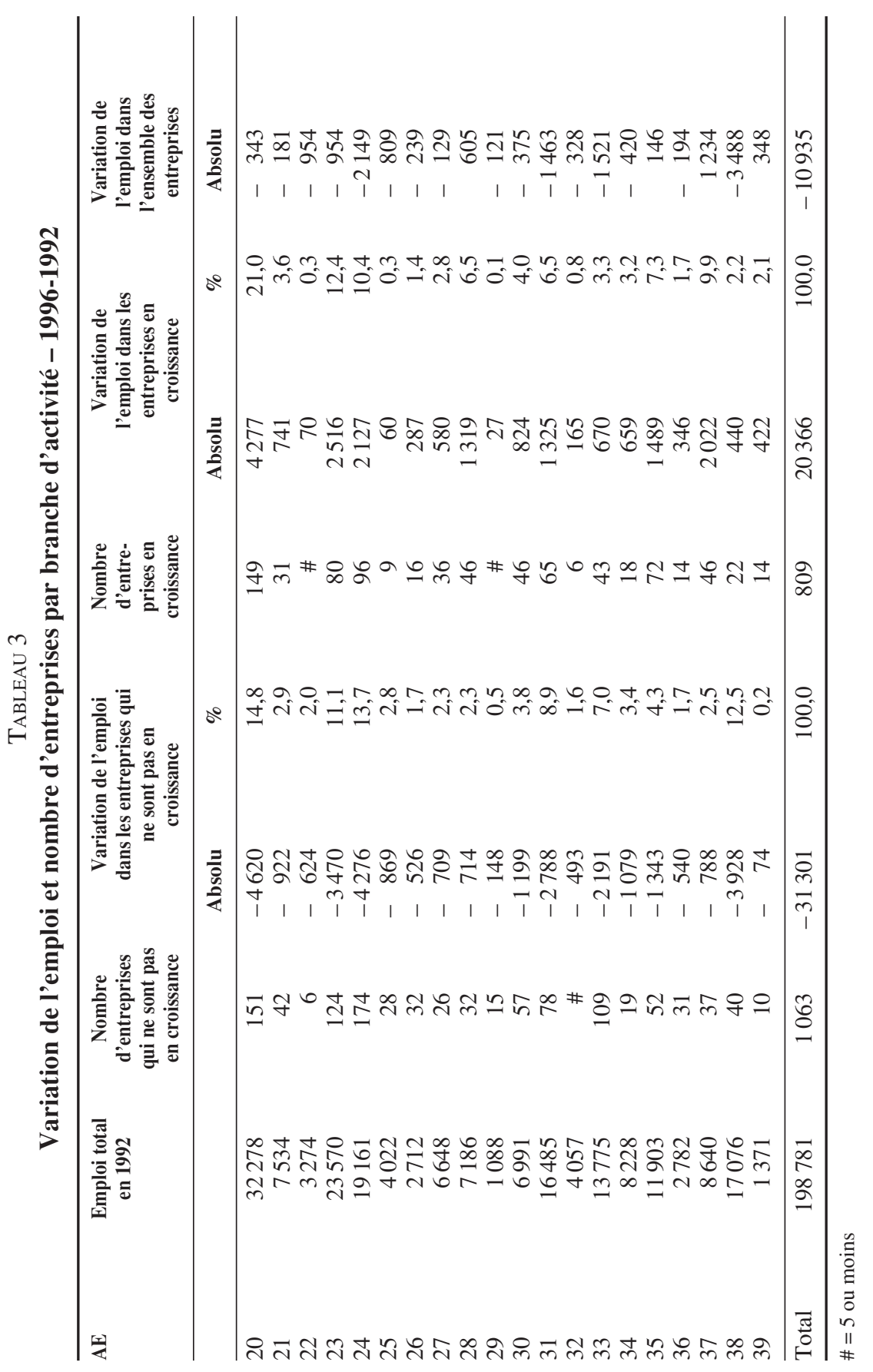

Revue internationale P.M.E., vol. 14, n ${ }^{\text {os }} 3-4,2001$ 


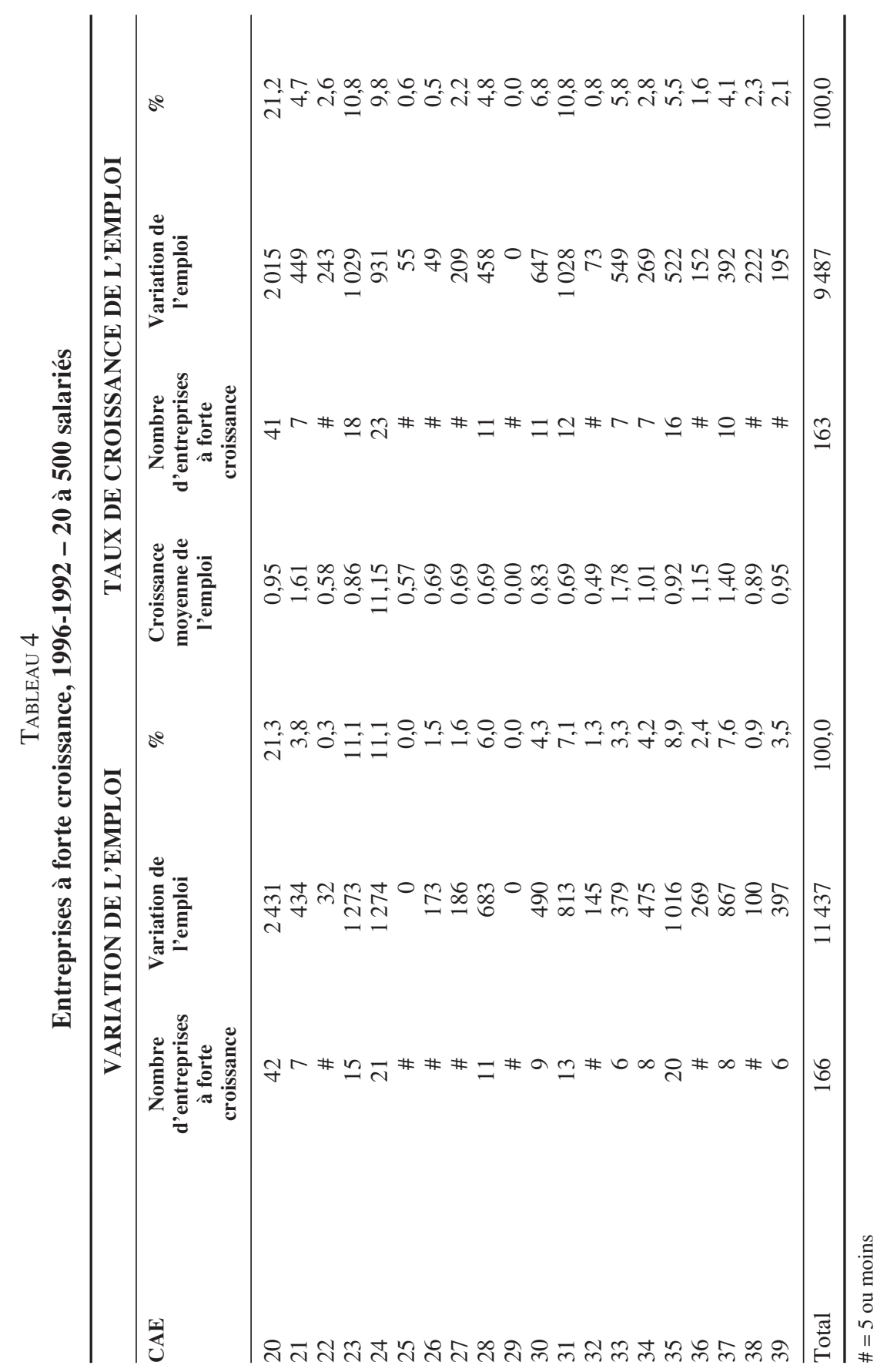

Revue internationale P.M.E., vol. 14, $\mathrm{n}^{\text {os }}$ 3-4, 2001 


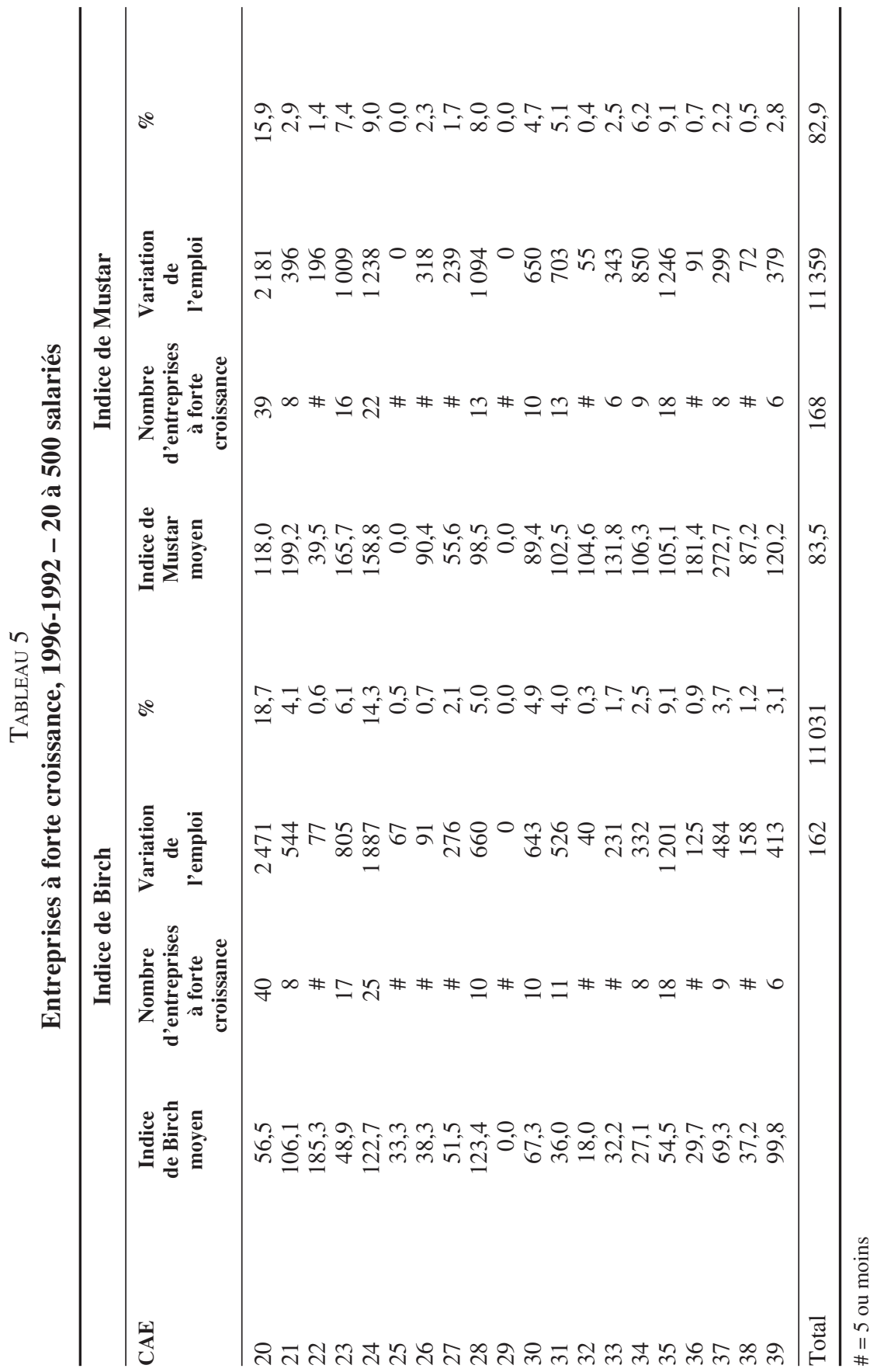

Revue internationale P.M.E., vol. 14, n ${ }^{\text {os }} 3-4,2001$ 


\section{Bibliographie}

AUDRETSCH, D. (1995), Innovation and Industry Evolution, Cambridge, MIT-Press.

BIRCH, D. (1981), «Who creates jobs?», The Public Interest 65.

BIRCH, D. (1987), Job Generation in America, New York, Free Press.

CARree, M. et L. Klomp (1996), «Small business and job creation : a comment», Small Business Economics, vol. 8, p. 317-332.

DAVIDSSON, P., L. LINDMARK et C. OLOFSSON (1998), « The extent of overestimation of small firm job creation : an empirical examination of the regression bias », Small Business Economics, vol. 11, p. 87-100.

DAVIS, J.S., J. HALTIWANGER et S. SCHUH (1996), «Small business and job creation: dissecting the myth and reassessing the facts », Small Business Economics, vol. 8, p. 297-315.

Droucopoulos, V. et S. THOMADAKIS, (1993), « The share of small and medium-size enterprise in Greek manufacturing », Small Business Economics, vol. 5, p. 187-196.

DunNe, P. et A. Hughes (1994), «Age, size, growth and survival: UK companies in the 1980s », The Journal of Industrial Economics, vol. XLII, nº 2, p. 115-140.

Dunne, P., M. Roberts et L. SAMUELSON (1989), «The growth and failure of U.S. manufacturing plants », Quarterly Journal of Economics, vol. 104, p. 671-698.

EVANS, D. (1987a), «The relationship between firm growth, size and age : estimates for 100 manufacturing industries », Journal of Industrial Economics, vol. 35, p. 567-581.

Evans, D. (1987b), «Tests of alternative theories of firm growth », Journal of Political Economy, vol. 95, p. 657-674.

FotOPOUlOS, G. et N. SPENCE (1998), « Accounting for net entry into Greek manufacturing by establishments of varying size », Small Business Economics, vol. 11, p. 125-144.

GREENE, W.H. (1997), Econometric Analysis, Englewood Cliffs, NJ, Prentice-Hall.

HALL, B. (1987), «The relationship between firm size and firm growth in the U.S.: manufacturing sector », Journal of Industrial Economics, vol. 35, p. 583-606.

KIRCHHOFF, B.A. et P.G. GreENE (1998), «Understanding the theoretical and empirical content of critiques of US job creation research », Small Business Economics, vol. 10, p. $153-169$.

OCDE (1997), High-Growth SME's and Employment: Assessment of Best Practice Policies, DSTI/IND/PME(97)9.

OCDE (2002), High-Growth SME's (à paraître).

STOREY, D.J. (1994), Understanding the Small Business Sector, Londres, Routledge.

STOREY, D.J. (1997), The Ten Percenters, Londres, Deloitte \& Touche.

ZARALIS, G. (1999), «High-growth firms and employment : country study of Greece», DSTI/IND/PME(99)8, OECD.

Revue internationale P.M.E., vol. 14, nos 3-4, 2001

(C) 2002 - Presses de l'Université du Québec

Édifice Le Delta I, 2875, boul. Laurier, bureau 450, Sainte-Foy, Québec G1V 2M2 • Tél. : (418) 657-4399 - www.puq.uquebec.ca

Tiré de : Revue internationale P.M.E., vol. 14, nos 3-4, sous la direction de Pierre-André Julien. 